Mathematical Research Letters 1, 35-43 (1994)

\title{
NOYAU DE LA CHALEUR D'OPÉRATEURS ELLIPTIQUES COMPLEXES
}

\author{
Pascal Auscher, Alan McIntosh, and Philippe Tchamitchian
}

\begin{abstract}
A ватRAст. We study the heat kernel of second order elliptic operators in divergence form with complex non-smooth bounded coefficients on $\mathbb{R}^{n}$. We obtain Gaussian bounds without further assumption if $n \leq 2$, and when the principal part has Hölder continuous coefficients if $n \geq 3$. Some applications are discussed.
\end{abstract}

\section{Estimations gaussiennes}

Les opérateurs que nous considérons sont de la forme

$$
\mathcal{L}=-\partial_{x_{i}}\left(a_{i j}(x) \partial_{x_{j}}\right)=-\operatorname{div}(A \nabla),
$$

où $a_{i j}(x) \in L^{\infty}\left(\mathbb{R}^{n}\right)$ est à valeurs complexes. On suppose qu'il existe $\delta>0$ tel que la matrice $A(x)=\left(a_{i j}(x)\right)_{1 \leq i, j \leq n}$ vérifie presque partout la condition d'ellipticité (appelée accrétivité):

$$
\forall \xi \in \mathbb{C}^{n} \quad \operatorname{Re} a_{i j}(x) \xi_{i} \bar{\xi}_{j} \geq \delta|\xi|^{2}
$$

ainsi que

$$
\forall \xi, \eta \in \mathbb{C}^{n} \quad\left|a_{i j}(x) \xi_{i} \bar{\eta}_{j}\right| \leq \delta^{-1}|\xi||\eta|
$$

Grâce à la formulation variationnelle, $\mathcal{L}$ est défini comme un opérateur maximal-accrétif sur $L^{2}\left(\mathbb{R}^{n}\right)$ et à ce titre, il engendre un semi-groupe de contraction $e^{-t \mathcal{L}}, t>0$. Notre objectif est d'étudier le noyau de la chaleur, $K_{t}(x, y)$, défini a priori comme le noyau-distribution de $e^{-t \mathcal{L}}$ et d'obtenir des estimations gaussiennes. Ces estimations sont bien connues si $A$ est à coefficients réels et dues à Aronson [Ar]. D'autres preuves avec une hypothèse de symétrie sont dues à Fabes et Stroock [FS] et Davies. L'étude du noyau de la chaleur est aussi liée à la géométrie des groupes et des variétés

Received October 15, 1993. 
(nous renvoyons à [Da, R, VSC] et leur bibliographie). Les arguments reposent de manière essentielle sur le fait que les coefficients sont réels (et dans une certaine mesure sur l'hypothèse de symétrie) via le principe du maximum, les inégalités de Harnack paraboliques ou les inégalités logarithmiques; ils ne s'adaptent pas au cas des coefficients complexes et il faut donc d'autres méthodes. Une de nos motivations pour étudier le cas des coefficients complexes est la conjecture de Kato sur le domaine de la racine carrée de $\mathcal{L}$.

Les hypothèses que nous faisons sont les suivantes. Si $n \leq 2$, on suppose que $A$ ne vérifie que les conditions (2) et (3). Si $n \geq 3$, outre (2) et (3), on suppose que les $a_{i j} \in C^{r}$, l'espace de Hölder d'exposant $r$ avec $0<r<1$. Pour une fonction $f$ définie sur $\mathbb{R}^{n}$, on désignera par $|f|_{r}$ la plus petite constante $C$ telle que $|f(x)-f(y)| \leq C|x-y|^{r}$ pour tous $x, y$. On pose $|A|_{r}=\sup \left|a_{i j}\right|_{r}$. Pour simplifier l'énoncé, on suppose que $r=0$ si $n \leq 2$ et l'on pose $|A|_{0}=0$. Avec ces hypothèses on a le résultat suivant.

\section{Théorème 1.}

(i) estimations ponctuelles : pour tout $t>0, K_{t}(x, y)$ est une fonction continue et il existe des constantes positives $C=C(\delta, n, r), \alpha=$ $\alpha(\delta, n)>0$ et $M=M(n)$ telles que pour tous $t>0$ et $x, y \in \mathbb{R}^{n}$,

$$
\left|K_{t}(x, y)\right| \leq C \frac{\left(1+|A|_{r}^{1 / r} t^{1 / 2}\right)^{M}}{t^{n / 2}} \exp \left\{-\frac{\alpha|x-y|^{2}}{t}\right\}
$$

où $M=0$ si $n \leq 2$.

(ii) régularité si $n=1$ : pour tout $t>0, K_{t}(x, y)$ est lipschitzienne en ses deux arguments, et $t^{1 / 2} \partial_{x} K_{t}(x, y)$ et $t^{1 / 2} \partial_{y} K_{t}(x, y)$, ainsi que $t \partial_{x} \partial_{y} K_{t}(x, y)$, vérifient presque partout l'inégalité (4) avec $n=1$ et $M=0$.

(iii) régularité si $n=2$ : il existe $\eta=\eta(\delta) \in] 0,1]$ tel que pour tout $t>0, K_{t}(x, y)$ soit une fonction höldérienne d'exposant $\eta$ et l'on a

$$
\begin{aligned}
& \left|K_{t}(x, y)-K_{t}(x+h, y)\right|+\left|K_{t}(x, y)-K_{t}(x, y+h)\right| \\
& \leq C \frac{\left(1+|A|_{r}^{1 / r} t^{1 / 2}\right)^{M}}{t^{n / 2}}\left(\frac{|h|}{t^{1 / 2}+|x-y|}\right)^{\eta} \exp \left\{-\frac{\alpha|x-y|^{2}}{t}\right\}
\end{aligned}
$$

lorsque $2|h| \leq t^{1 / 2}+|x-y|$ avec $M=0, C=C(\delta, n, r), \alpha=$ $\alpha(\delta, n)>0$ et $n=2$.

(iv) régularité si $n \geq 3$ : pour tout $\eta \in] 0, r\left[\right.$ et tout $t>0, K_{t}(x, y)$ est de classe $\mathcal{C}^{1+\eta}$ en chaque variable. De plus, $t^{1 / 2} \nabla_{x} K_{t}(x, y)$ et $t^{1 / 2} \nabla_{y} K_{t}(x, y)$ vérifient les inégalités (4) et (5) pour une valeur finie de $M$. 
Corollaire. Sous les hypothèses ci-dessus, on a

$$
\left\|e^{-t \mathcal{L}}\right\|_{\infty, \infty} \leq C\left(1+|A|_{r}^{1 / r} t^{1 / 2}\right)^{M}
$$

où les constantes sont celles du point (i).

On a posé $\|T\|_{q, p}$ la norme d'un opérateur borné de $L^{p}$ dans $L^{q}$. Le semigroupe est donc borné sur $L^{\infty}$ mais nous ne savons pas s'il est équicontinu sur $L^{\infty}$ lorsque $n \geq 3$, à la différence du cas des coefficients réels où $e^{-t \mathcal{L}}$ est un semi-groupe de contraction sur tous les $L^{p}$.

\section{Remarques.}

(1) Les méthodes que nous employons conviennent également aux perturbations de $\mathcal{L}$ par des termes d'ordre inférieur, soit

$$
\mathcal{L}^{\prime}=-\partial_{x_{i}}\left(a_{i j}(x) \partial_{x_{j}}+a_{i, n+1}(x)\right)+a_{n+1, j}(x) \partial_{x_{j}}+a_{n+1, n+1}(x),
$$

les indices $i, j$ parcourant $1, \cdots, n$, et on suppose que la matrice $\left(a_{k \ell}(x)\right)_{1 \leq k, \ell \leq n+1} \equiv \mathcal{A}(x)$ vérifie les inégalités (2) et (3) (où $n$ est remplacé par $n+1)$. La partie principale de $\mathcal{L}^{\prime}$ est soumise aux mêmes hypothèses que ci-dessus mais aucune autre condition n'est imposée sur les coefficients restants. Le noyau, $K_{t}^{\prime}(x, y)$, de $e^{-t \mathcal{L}^{\prime}}$ vérifie alors les mêmes propriétés à l'exception de (iv) où l'exposant de régularité n'est plus que $1-\varepsilon$, pour tout $\varepsilon>0$. De plus, les membres de droite dans (4) et (5) peuvent être multipliés par $e^{-\delta t / 2}$, $\delta$ étant la constante de $(2)$ pour $\mathcal{A}(x)$, ce qui compense le facteur polynomial $\left(1+|A|_{r}^{1 / r} t^{1 / 2}\right)^{M}$. Signalons cependant que la régularité lipschitzienne dans (ii) est plus délicate à obtenir pour $K_{t}^{\prime}(x, y)$ à cause des termes d'ordre 1 .

(2) Revenons à l'enoncé du théorème. Lorsque $n \geq 3$, l'hypothèse de régularité se paye dans (4) et (5) par le facteur polynomial $\left(1+|A|_{r}^{1 / r} t^{1 / 2}\right)^{M}$. Ce facteur s'obtient en suivant pas-à-pas la démonstration et est donc meilleur qu'un facteur exponentiel que l'on obtiendrait en écrivant $e^{-t \mathcal{L}}=e^{\varepsilon t} e^{-t(\mathcal{L}+\varepsilon)}$ et en appliquant le théorème à $\mathcal{L}+\varepsilon$. Noter que si $n=2$ et si $A(x) \in C^{r}, r>0$, alors la conclusion de (iv) est également valable et les estimations (4) et (5) sur les dérivées premières de $K_{t}(x, y)$ sont valides (mais nous ne savons pas si l'on peut prendre $M=0$ ). En dimension 1 , les points (i) et (ii) sont mentionnés dans [AT] sans démonstration. Une dernière remarque est que le terme gaussien dans ces inégalités ne dépend pas de la régularité des coefficients. 
Passons à la méthode utilisée, qui fournit à la fois régularité et estimations ponctuelles (i); nous nous concentrons uniquement sur ces dernières. Usuellement on déduit du noyau de la chaleur des informations sur la résolvante ou même sur les fonctions de $\mathcal{L}$ (voir [Da]). Nous allons procéder de façon inverse. Il y a trois ingrédients: une intégrale de contour avec un argument de renormalisation, un théorème de régularité et un résultat de perturbation.

Tout d'abord, il faut un algorithme pour calculer le semi-groupe. On utilise la formule

$$
e^{-t \mathcal{L}}=\frac{(m-1) !}{2 \pi i t^{m-1}} \int_{\gamma} e^{t \lambda}(\lambda+\mathcal{L})^{-m} d \lambda
$$

que l'on obtient formellement en intégrant par parties la formule de Cauchy $(m=1)$. L'intégrale ne dépend pas du choix de $\gamma$ pourvu qu'elle converge. On a donc

$$
K_{t}(x, y)=\frac{(m-1) !}{2 \pi i t^{m-1}} \int_{\gamma} e^{t \lambda} R_{\lambda}^{(m)}(x, y) d \lambda
$$

où $R_{\lambda}^{(m)}(x, y)$ est le noyau de $(\lambda+\mathcal{L})^{-m}$ et où $\gamma$ se compose de deux demidroites $\lambda=r e^{ \pm i \theta}, r \geq R$ et de l'arc de cercle $\lambda=R e^{i \omega},|\omega| \leq \theta$. On peut choisir $\theta \in] \pi / 2, \pi\left[\right.$ et on prend $R=\sup \left(\beta|x-y|^{2} t^{-2}, t^{-1}\right)$ où $\beta$ est une constante positive à déterminer.

L'intérêt d'utiliser une itérée de la résolvante est que les propriétés de régularité et de décroissance sont meilleures pour les itérées que pour la résolvante même. Nous avons le choix de l'entier $m$ mais pour récupérer une estimation gaussienne sur $K_{t}(x, y)$ à partir de $(7)$ il faut démontrer une estimation exponentielle sur $R_{\lambda}^{(m)}(x, y)$ pour $\lambda$ dans le secteur $|\arg \lambda| \leq \theta$.

Le deuxième ingrédient est un résultat de régularité sur les opérateurs du type (6).

Proposition 1. Soit $\mathcal{L}^{\prime}$, défini par (6), vérifiant les hypothèses de la remarque 1. Alors il existe un entier $m=m(n) \geq 1$ tel que $\mathcal{L}^{\prime-m}: L^{1} \rightarrow L^{\infty}$ est borné avec $\left\|\mathcal{L}^{\prime-m}\right\|_{\infty, 1}=C(\delta, n, r)\left(1+|A|_{r}^{1 / r}\right)^{M(n)}$.

Rappelons que l'on a posé $|A|_{r}=0$ si $r=0$, ce qui s'applique en dimensions 1 et 2 . Ce type de résultat itératif est classique si les coefficients sont très réguliers et remonte aux travaux de Nirenberg $[\mathrm{N}]$, de Nelson et Stinespring [NS] et aussi d'Agmon [Ag]: on utilise les théorèmes de régularité pour les équations elliptiques dans la chaîne des espaces de Sobolev $W^{s, 2}$ et les inclusions de Sobolev $W^{s, 2} \subset L^{\infty}$ si $s>n / 2$. Mais si les coefficients ne sont que mesurables, la marge de manoeuvre est parmi les Sobolev $W^{s, p}$ 
pour $-1 \leq s \leq 1$ et $1 \leq p \leq \infty$. On peut alors essayer d'augmenter l'indice $p$ en itérant la résolvante de façon à conclure avec l'inclusion $W^{1, p} \subset L^{\infty}$ si $p>n$.

Pour $n=1, m=1$ convient trivialement. Si $n=2$ on peut choisir $m=2$ en observant que $\mathcal{L}^{\prime}$ est inversible de $W^{1, p}$ sur $W^{-1, p}$ si $|p-2|$ est petit par perturbation analytique du cas $p=2$. Pour $n \geq 3$, la proposition repose sur le résultat de régularité suivant dû à $\mathrm{M}$. Taylor $[\mathrm{T}]$ pour la partie principale $\mathcal{L}=-\operatorname{div}(A \nabla)$ de $\mathcal{L}^{\prime}$.

Lemme 1. Si $A$ vérifie (2) et (3), les conditions $\operatorname{div}(A \nabla u)=f, u \in W^{1, p}$, $f \in W^{-1, q}, A \in C^{r}$ avec $1<p<q<+\infty$ et $r>0$ impliquent $u \in W^{1, q}$.

Une application répétée de ce lemme et des inclusions de Sobolev montre qu'il existe $k$ tel que $\mathcal{L}^{\prime-k}$ envoie $L^{2}$ dans $L^{\infty}$. Comme $\mathcal{L}^{\prime} *$ est du même type que $\mathcal{L}^{\prime}$, on a également que $\mathcal{L}^{\prime-k}$ envoie $L^{1}$ dans $L^{2}$ par dualité, et donc $\mathcal{L}^{\prime-2 k}$ envoie $L^{1}$ dans $L^{\infty}$.

Le troisième ingrédient est la méthode de Davies [Da] pour obtenir la décroissance à l'infini par des perturbations exponentielles laissant stable la classe des opérateurs du type $\mathcal{L}^{\prime}$.

Lemme 2. Soit $\mathcal{L}^{\prime}$ comme ci-dessus. Alors il existe $\alpha=\alpha(\delta, n)>0$ et une constante positive $C=C(\delta, n, r)\left(1+|A|_{r}^{1 / r}\right)^{M(n)}$ tels que si $m$ est l'entier de la proposition 1 ,

$$
\left|R^{\prime(m)}(x, y)\right| \leq C \exp \{-\alpha|x-y|\} .
$$

On a posé $R^{\prime(m)}(x, y)$ le noyau de $\mathcal{L}^{\prime-m}$. En appliquant ce lemme à $R_{\lambda}^{(m)}(x, y)$ et à l'aide d'une renormalisation par changement d'échelle, on obtient

$$
\begin{aligned}
\left|R_{\lambda}^{(m)}(x, y)\right| \leq C(\delta, n, r)\left(\left(1+|A|_{r}^{1 / r}|\lambda|^{-1 / 2}\right)^{M(n)}\right. & |\lambda|^{n / 2-m} \exp \left\{-\alpha|\lambda|^{1 / 2}|x-y|\right\}
\end{aligned}
$$

pour tout $x, y$ et $\lambda$ dans un secteur $|\arg \lambda| \leq \theta$, le choix de $\theta>\pi / 2$ ne dépendant que de $\delta$ et $n$. La formule (7) donne l'estimation gaussienne cherchée. On obtient la régularité suivant le même ordre d'idées.

Cette méthode peut se révéler efficace même si les opérateurs considérés sont auto-adjoints; elle est utilisée à nouveau dans [AER]. Il est naturel de se demander si l'on peut généraliser le théorème 1 au cas des coefficients mesurables lorsque $n \geq 3$. En utilisant d'autres théorèmes de régularité, cette méthode conduit à une nouvelle démonstration du résultat d'Aronson lorsque $A$ est réelle (symétrique ou non) [Au]. 


\section{Quelques applications}

Commençons par un corollaire de la démonstration du point (ii) du théorème 1.

Proposition 2. On suppose $n=1$ et on se donne $\mathcal{L}^{\prime} d u$ type (6) avec les hypothèses de la remarque 1 . Alors pour $1 \leq p \leq \infty, \mathcal{L}^{\prime-1}$ admet une extension continue de $W^{-1, p}$ dans $W^{1, p}$ et de $L^{p}$ dans $W^{1, \infty}$.

En dimension $n \geq 2$ l'inversibilité de $\mathcal{L}^{\prime}$ de $W^{1, p}$ sur $W^{-1, p}$ n'est vraie en général que si $p$ est voisin de 2 et les solutions de $\mathcal{L}^{\prime} u=f, f \in L^{p}$, ne sont pas lipschitziennes. C'est donc un résultat spécifique à la dimension 1. Notons que $-d_{x} a d_{x}$, la partie principale de $\mathcal{L}^{\prime}$, est par définition un isomorphisme entre les espaces de Sobolev homogènes $\dot{W}^{1, p}$ et $\dot{W}^{-1, p}$. Pour $\mathcal{L}^{\prime}$, la difficulté réside dans les termes d'ordre 1 .

Continuons par une application à la conjecture de Kato.

Théorème 2. Soit $\mathcal{L}$ donné par (1) et vérifiant (2) et (3). On suppose que $n \geq 2$ et que $A \in C^{r}, 0<r<1$. Alors le domaine de $\mathcal{L}^{1 / 2}$ coïncide avec $W^{1,2}$. De plus,

$$
\mathcal{L}^{1 / 2}=T_{i} a_{i j} \partial_{x_{j}}+R,
$$

où $R$ est borné sur $L^{2}$ et $T_{i}$ est un opérateur de Calderón-Zygmund. En outre, si $n=2, R$ est borné sur tous les $L^{p}, 1 \leq p \leq \infty$ et par suite, $\mathcal{L}^{1 / 2}$ admet une extension continue de $W^{1, p}$ dans $L^{p}$ pour $1<p<\infty$.

La caractérisation du domaine de $\mathcal{L}^{1 / 2}$ sous ces hypothèses est due à l'un des auteurs [McI1]. Une description de $\mathcal{L}^{1 / 2}$ en termes d'opérateurs de Calderón-Zygmund a été obtenue par Alexopoulos [Al] qui suppose, en outre, que les coefficients sont réels et périodiques. En dimension 1, aucune condition de régularité n'est nécessaire pour que le domaine de $\mathcal{L}^{1 / 2}$ coïncide avec $W^{1,2}[\mathrm{CMcM}]$ et on trouve dans $[\mathrm{AT}]$ une description optimale en termes d'opérateurs de Calderón-Zygmund.

Pour établir (8), on part de

$$
\begin{aligned}
& \mathcal{L}^{1 / 2}=\frac{1}{\sqrt{\pi}} \int_{0}^{+\infty} e^{-t \mathcal{L}} \mathcal{L} \frac{d t}{t^{1 / 2}} \\
& =-\frac{1}{\sqrt{\pi}} \int_{0}^{1} e^{-t \mathcal{L}} t^{1 / 2} \partial_{x_{i}} \frac{d t}{t} a_{i j} \partial_{x_{j}}+\frac{1}{\sqrt{\pi}} \int_{1}^{+\infty} e^{-t \mathcal{L}} t \mathcal{L} \frac{d t}{t^{3 / 2}} \\
& \equiv T_{i} a_{i j} \partial_{x_{j}}+R \text {. }
\end{aligned}
$$

Les estimations (4) et (5) pour $t^{1 / 2} \partial_{y_{i}} K_{t}(x, y), t \leq 1$, impliquent que le noyau de $T_{i}$ est du type Calderón-Zygmund et la continuité de $T_{i}$ sur $L^{2}$ 
s'obtient avec le théorème $T(1)$ de David et Journé $[\mathrm{DJ}]$ en remarquant que $T_{i}(1)=T_{i}^{*}(1)=0$. L'intégrale définissant $R$ converge normalement dans $\mathcal{L}\left(L^{2}\right)$ car sup $\left\|t \mathcal{L} e^{-t \mathcal{L}}\right\|_{2,2}<+\infty$ par le calcul fonctionnel. De plus, si $n=2$ le noyau de $t \mathcal{L} e^{-t \mathcal{L}}$ vérifie également (4) avec $M=0$ et donc le noyau de $R$ se majore par $C(1+|x-y|)^{-3}$, d'où le résultat.

Remarque. L'opérateur $R$ n'apparaît pas dans [Al] du fait d'un meilleur contrôle des estimations gaussiennes pour $t^{1 / 2} \partial_{y_{i}} K_{t}(x, y)$ lorsque $t \rightarrow+\infty$.

La dernière application concerne le calcul fonctionnel pour l'opérateur défini par (1). Cet opérateur étant maximal-accrétif, il possède un calcul fonctionnel $H^{\infty}$ sur $L^{2}$. Ceci veut dire que l'on a l'estimation

$$
\|b(\mathcal{L})\|_{2,2} \leq c_{\mu}\|b\|_{\infty}
$$

pour toute fonction $b \in H^{\infty}\left(S_{\mu}^{0}\right)$, où $S_{\mu}^{0}$ est le secteur angulaire $\{z \in \mathbb{C}$ : $|\arg z|<\mu\}$, et $\mu>\omega \equiv \sup \left\{\left|\arg a_{i j}(x) \zeta_{i} \bar{\zeta}_{j}\right|: \zeta \in \mathbb{C}^{n}, x \in \mathbb{R}^{n}\right\}[\mathrm{McI} 2]$.

Une question naturelle consiste à savoir si $b(\mathcal{L})$ est un opérateur de Calderón-Zygmund, ce qui implique la continuité sur $L^{p}, 1<p<+\infty$. Dans $[\mathrm{Du}]$, cela est démontré lorsque la matrice $A$ est symétrique réelle à coefficients mesurables en utilisant des estimations sur la résolvante. Cela peut également se faire en utilisant le noyau de la chaleur.

Théorème 3. Soit $\mathcal{L}$ vérifiant les hypothèses du théorème 1 . Soit $b \in$ $H^{\infty}\left(S_{\mu}^{0}\right)$, telle que

$$
|b(\zeta)| \leq c_{0}\left(1+|A|_{r}^{1 / r}|\zeta|^{-1 / 2}\right)^{-M}, \zeta \in S_{\mu}^{0}
$$

où $M$ est la meilleure constante dans (4) et $(5)$. Alors $b(\mathcal{L})$ est un opérateur de Calderón-Zygmund. De plus, on a l'estimation

$$
\|b(\mathcal{L})\|_{p, p} \leq c\left(\mu, n, p, c_{0}\right)\|b\|_{\infty}
$$

si $1<p<\infty$.

En dimensions 1 et 2 on a $M=0$ et le calcul fonctionnel $H^{\infty}$ se prolonge aux espaces $L^{p}$ en toute généralité si les coefficients sont complexes et mesurables. Si $n \geq 3$, la condition (9) exprime que la décroissance de $b(\zeta)$ à l'origine doit compenser le terme polynomial dans (4).

La méthode repose sur la formule de representation

$$
b(\mathcal{L})=\frac{1}{2 \pi} \lim _{\delta \rightarrow 0} \int_{\gamma} e^{i z \mathcal{L}} \check{b}(z+i \delta) d z
$$


où $\gamma$ est constitué des deux demi-droites $r e^{i(\pi+\theta)}$ et $r e^{-i \theta}, \omega<\theta<\mu$, $r>0$ (on a donc $|\arg i z| \leq \pi / 2-\theta<\pi / 2-\mu$ ). La fonction $\check{b}$ désigne la transformée de Fourier inverse de $b$ (en posant $b(\zeta)=0$ si $\operatorname{Re} \zeta<0$ ), prolongée holomorphiquement dans le secteur $i S_{\frac{\pi}{2}+\mu}^{0}$. En suivant [McQ], on démontre que

$$
|\check{b}(z)| \leq c_{\nu}|z|^{-1}\left(1+|A|_{r}^{1 / r}|z|^{1 / 2}\right)^{-M}
$$

sur tout secteur restreint $i S_{\frac{\pi}{2}+\nu}^{0}, \nu<\mu$.

Il reste à obtenir un prolongement holomorphe $S_{z}$ du semi-groupe dans le secteur $|\arg z|<\frac{\pi}{2}-\mu$. On peut vérifier que (4) et (5) sont valides à condition de remplacer $t$ par $|z|$.

On déduit de (10) par des estimations directes que le noyau de $b(\mathcal{L})$ est un noyau de type Calderón-Zygmund avec estimations Höldériennes d'exposant $\eta>0$.

Signalons pour finir que des arguments similaires montrent que si $\mathcal{L}^{\prime}$ est défini par (6) avec les hypothèses de la remarque 1 alors $b\left(\mathcal{L}^{\prime}\right)$ est un opérateur de Calderón-Zygmund pour toute fonction $b \in H^{\infty}$ définie sur un secteur $S_{\mu}^{0}$ convenable.

\section{Remerciements}

Les détails de cette note seront publiés ultérieurement. Parties de ce travail ont été réalisées à l'Université de Saint-Jérôme et à Macquarie University et le premier auteur remercie ces deux institutions et l'Australian Research Council pour leurs supports financiers. Le second auteur remercie également l'Université de Rennes où cette collaboration a débuté.

\section{References}

[Ag] S. Agmon, Lectures on elliptic boundary value problems, Van Nostrand Mathematical Studies 2, D. van Nostrand Company, Inc, Princeton, 1965.

[Al] G. Alexopoulos, La conjecture de Kato pour les opérateurs différentiels elliptiques à coefficients périodiques, C. R. Acad. Sci. Paris, Série 1312 (1991), 263-266.

[Ar] D. Aronson, Bounds for fundamental solutions of a parabolic equation, Bull. Amer. Math. Soc. 73 (1967), 890-896.

[Au] P. Auscher, Regularity theorems and heat kernels for elliptic operators (to appear).

[AER] P. Auscher, A. F. M. ter Elst, and D. W. Robinson, On positive Rockland operators, preprint CMA-MR20-93, the Australian National University.

[AT] P. Auscher and Ph. Tchamitchian, Une nouvelle approche de la conjecture de Kato et équations elliptiques complexes en dimension 2, preprint, Université de Rennes 1. 
[CMcM] R. Coifman, A. McIntosh, and Y. Meyer, L'intégrale de Cauchy définit un opérateur borné sur $L^{2}(\mathbb{R})$ pour les courbes lipschitziennes, Ann. Math. 116 (1982), 361-387.

[DJ] G. David and J.-L. Journé, A boundedness criterion for generalized CalderónZygmund operators, Ann. Math 120 (1984), 371-398.

[Da] E. B. Davies, Heat kernels and spectral theory, Cambridge Univ. Press, 1992.

[Du] X. T. Duong, $H^{\infty}$ functional calculs of elliptic partial differential operators in $L^{p}$ spaces, $\mathrm{PhD}$ thesis, Macquarie University, 1990.

[FS] E. B. Fabes and D. W. Stroock, A new proof of Moser's parabolic Harnack inequalities via the old ideas of Nash, Arch. Rat. Mech. Anal. 96 (1986), 327338 .

[McI1] A. McIntosh, Square roots of elliptic operators, J. Funct. Anal. 61 (1985), 307-327.

[McI2] A. McIntosh, Operators which have an $H^{\infty}$-functional calculus, Miniconference on Operator Theory and Partial Differential Equations, Proceedings of the Centre for Mathematical Analysis, ANU, Canberra, vol. 14, 1986, pp. 210-231.

[McQ] A. McIntosh and T. Qian, Convolution singular integral operators on Lipschitz curves, Proceedings of the Special Year on Harmonic Analysis at Nankai Institute of Mathematics, Tianjin, China, Lecture Notes in Mathematics, vol. 1494, Springer-Verlag, Berlin, 1991, pp. 142-162.

[NS] E. Nelson and W. F. Stinespring, Representation of elliptic operators in an enveloping algebra, Amer. J. of Math. 81 (1955), 547-560.

[N] L. Nirenberg, Remarks on strongly elliptic partial differential operators, Comm. Pure \& Appl. Math. 8 (1955), 649-675.

[R] D. W. Robinson, Elliptic operators and Lie groups, Oxford Mathematical Monographs, Oxford University Press, Oxford, 1991.

[T] M. E. Taylor, Pseudodifferential operators and non-linear P.D.E., Birkhauser,, Boston, 1992.

[VSC] N. T. Varopoulos, L. Saloff-Coste, and T. Coulhon, Analysis and geometry on groups, Cambridge tracts in mathematics, vol. 100, Cambridge University Press, 1992.

C N R S, iR M A R, Univerité de Rennes I, Campus de Beaulieu, 35042 Rennes Cedex, France and M athematics Department, Brown University, Providence, R I 02912 , USA

E-mail address: auscher@roland.univ-rennes1.fr and auscher@gauss.math.brown.edu

Department of M Athematics, Macquarie University, N SW 2109 , Australia E-mail address: alan@macadam.mpce.mq.edu.au

Faculté des Sciences and Techniques de Saint Jérôme, 13397 M arseille CEDEX 20 , FRANCE

E-mail address: tchamphi@frmrs11.bitnet 